\title{
DAMPING EFFECT IN THE BEAM ENVELOPE AMPLITUDE OSCILLATIONS IN MISMATCHED HIGH INTENSITY ION BEAMS
}

\author{
T. Clauser, V. Stagno, Dipartimento di Fisica di Bari, \\ V. Variale, INFN sez.di Bari, Via Amendola 173 Bari 70126, Italy
}

\begin{abstract}
Space-charge dominated beams can induce chaotic behavior on particle trajectories leading to halo formation on the beam spot. This causes particle losses along the beam transportation that must be minimized. The fractional losses must be kept below $10^{-7} / \mathrm{m}$. This is a very low threshold to check with standard multiparticle codes. To study this kind of problems a new particle simulation approach will be proposed in this paper in analogy with the single particle to core interaction model which is the most used calculation technique applied in these kind of phenomena. In particular, a damping effect in the amplitude oscillations of the beam envelope, for high intensity beam mismatched with a FODO cell, will be shown and discussed.
\end{abstract}

\section{INTRODUCTION}

In these last years, growing interest has been addressed, from the international scientific community, on the possible applications of high intensity ion beams. Among them, just as examples, we can mention the energy amplifier proposed by C. Rubbia [1] and the transmutations of radioactive waste [2]. However high intensity beam transport poses problems those need to be faced and solved. In particular, a halo formation has been observed around high intensity beams, during the transport, that leads to particle losses. For high current and energy beams the lost particles produce radio activation in the structures and the related radiation can damage the accelerator components. Furthermore the radioactivation makes the accelerator maintenance very difficult and expensive. Because of these problems, it becomes very important to study halo formation mechanism in the beam.

Multiparticle codes could be very helpful to this aim but it is very difficult to study this kind of phenomena by using the standard multiparticle codes because the lost particle fraction, along the transport, must be kept below $10^{-7} / \mathrm{m}$ [3]. This means that the code should use a number of particles of the order of $10^{+6}$, in the simulations, to appreciate this kind of lost fractions. This number is very high and very powerful calculators are needed to handle them. In fact, in these kinds of simulations, the space charge force effect, because of the high intensity beam used, becomes a very important issue.
A new calculation technique, called Particle Core Model (PCM) [4], has been introduced to study the halo formation without using of very powerful computers.

The PCM solves the beam envelope (or rms) equation for a continuous beam that is used as a model for the core of the beam. The core can be mismatched so that its radius, taken at the exit of the transport period cell, will oscillate. The halo particles are represented by test particles, which oscillate through the core, influenced by linear external focusing field and the non-linear space charge fields of the core. The model allows one to study the dynamics of the test particles.

Although the PCM calculations allow studying the halo formation, this is done by assuming some approximations and then their influence on the PCM results should be checked.

For that reason, a comparison test between the PCM results and the simulations of the multiparticle code PARMT, modified to allow a more direct comparison with the results of PCM calculations, has been carried out in ref. [5].

In that reference, a continuous beam has been transported through hundreds of FODO periodic cells. The comparison of the simulation results obtained either with the multiparticle code and PCM leaded to the following conclusions. PCM calculations seem unsuitable to study test particles taken near the beam core region in the case of high beam current. The discrepancies found between the multiparticle code and PCM results were mainly due to the following reason.

In PARMT simulations, the breathing mode oscillations, due to the mismatch of the input beam with the periodic cells, damp very quickly when the space charge is strong. In PCM calculations, a constant amplitude oscillation of the beam envelope is, instead, considered.

In this paper, an attempt to better understand the mechanism causing the fast damping of the beam breathing mode oscillations observed in ref. [5] will be done. Moreover, further simulations on the chaotic behavior originating, in some cases, in the test particle trajectories will be also shortly shown and discussed.

\section{SIMULATIONS}

The multiparticle code PARMT is a Monte Carlo program that can transport an ion beam through a system of optic elements by using the matrix method [6]. Being simulated a very high number of particles, PARMT uses a 
'Fast Poisson Solver' (FPS) technique to compute the space charge electric field of the ion beam.

To compare directly the PCM with the PARMT results some modifications have been carried out in the PARMT code. Mainly, two new features have been introduced in the program: 1) an input file with the initial test particles coordinates that can be read, if required, by the main program; 2) $\mathrm{N}$ output files (with $\mathrm{N}$ number of test particles), where the test particle phase space coordinates along the transport in the periodic cells $(\mathrm{Np})$ are stored.

The main input file parameters, used in the PARMT simulations of ref. [5], are here recalled. In that simulations we used FODO cell periods of length $\mathrm{L}=80$ $\mathrm{cm}$; transverse rms emittances, $\varepsilon_{\mathrm{x}}=\varepsilon_{\mathrm{y}}=0.25 \times 10^{-6} \mathrm{~m} \cdot \mathrm{r}$; single particle phase advance $\sigma_{0}=60.7^{\circ}$ and space charge parameter (as defined in ref [7]) $\xi \quad 4 \times 10^{-6}$ (corresponding to $\mathrm{I}=95 \mathrm{~mA}$ and $\beta=0.145$ ).
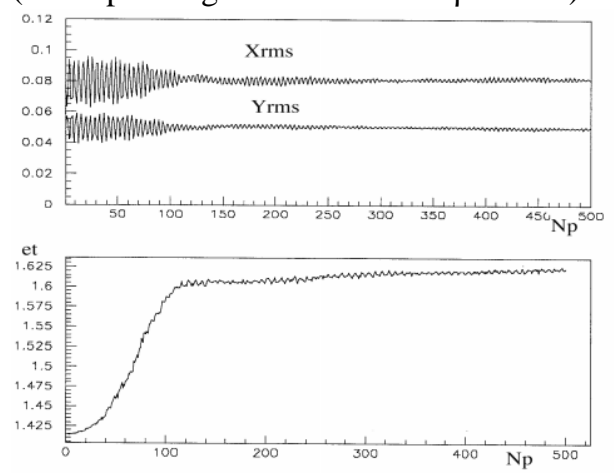

Fig.1Transverserms beam size values $v s$ cell period numbers $(\mathrm{Np})$ for the case $\sigma / \sigma_{0}=0.55$.

Moreover an odd mismatching of about $20 \%$ has been added on the initial beam size, in all the simulations.

The fast oscillation damping of the beam breathing mode found in ref.[5] is presented in fig.1. In that figure the related total emittance variation et, defined as $\mathrm{et}=\left(\varepsilon_{\mathrm{x}}{ }^{2} / \varepsilon_{\mathrm{x} 0}{ }^{2}+\varepsilon_{\mathrm{y}}{ }^{2} / \varepsilon_{\mathrm{y} 0}{ }^{2}\right)^{1 / 2}$, is also shown.

We think that the damping effect of the fig.1 can be ascribed to the Landau mechanism of stabilization that can occur when a very large number of oscillators, which are the same but for their natural frequency, are excited by an external force.

This mechanism is already used in accelerator physics to explain the Keil-Schnell stability criterion [8]. In fact, if one injects a very cool beam in accelerator machine, the microwave instability develops. The beam momentum spread blows up to a point when Landau damping gets strong enough to stabilize the unstable motion. The KeilShnell criterion gives the minimum energy spread, that is betatron frequency spread, for stability. The Landau damping is a stabilizing effect connected with the frequency spread [9].

To clarify, shortly, the Landau damping mechanism, consider a set of oscillators, with a frequency distribution $\mathrm{f}\left(\omega_{\beta}\right)$, coupled to a harmonic excitation of frequency $\omega$.
The response of the set to the excitation can be seen by the center-of-charge velocity of the set [10]:

$\langle\dot{y}\rangle=\hat{G} e^{-i \omega t}\left[\pi f(\omega)-i P V \int \frac{f(\omega)}{\omega_{\beta}-\omega} d \omega_{\beta}\right]$

where PV means Principal Value integral and $\hat{G}$ is the amplitude of the harmonic excitation.

This response has a real part for which the velocity and the excitations are in phase and an imaginary part, where they are out of phase, indicating no energy exchange. The real part term indicates that the beam absorbs energy from the exciting signal. Since the beam particles absorb energy this can lead to the damping of the coherent oscillation that is often called Landau damping. From the equation (1) it can be noticed that the real term is proportional to the density of the frequency distribution, $f\left(\omega_{\beta}\right)$, in the region of the excitation frequency $\omega$. Then a condition to obtain an effect of Landau damping is that the exciting frequency lies inside the incoherent frequency distribution. Furthermore, we have to add from ref. [10], that the equation (1) is only correct if the excitation has lasted for a long time. How long this time has to be depends on the shape of the frequency distribution $f\left(\omega_{\beta}\right)$ considered. If this distribution does not change significantly over a frequency range of $\Delta \omega_{\beta}$, around $\omega$, it is sufficient to excite for a time $\tau \gg>1 / \Delta \omega_{\beta}$. This means that all the oscillators with frequency inside $\Delta \omega_{\beta}$, in the time $\tau$, are still responding positively to the exciting force. Then to have a large period of interaction between the exciting force and the oscillators we need a large $\Delta \omega_{\beta}$. In conclusion, the Landau damping mechanism applies only if we have oscillators with an enough large frequency spectrum that cover the exciting frequency.

To interpret the behavior of fig. 1 we need to make some preliminary considerations.

The beam particles, in the reference particle system, can be seen as a set of oscillators that oscillate with their betatron frequency because of the focusing forces. When the beam particles have a certain momentum spread, because of the cromaticity, they will have also a betatron frequency spread. In general, this frequency spread tends to be very small, but when we have a space charge dominated beam $\left(\mathrm{Kr}^{2} / 4 \varepsilon>>1[11]\right.$, see below for the symbol meaning) it enlarges very much. The space charge tune depression $\sigma / \sigma_{0}$ in the case of the simulations shown in fig. 1 was 0.55 . This means that the most inner beam particles will still have a phase advance $\sigma_{\mathrm{o}}$ of $60.7^{\circ}$, that is, a betatron frequency $\omega_{\beta}$ of about $58 \mathrm{MHz}$, while the most external beam particles, filling the whole space charge force, will have a phase advance $\sigma=0.55\left(60.7^{\circ}\right)=33.4^{\circ}$, that is $\omega_{\beta}=31.6 \mathrm{MHz}$. On the other hand, when a space charge dominated beam is mismatched with the focusing periodic cell, the beam itself starts to oscillate in a breathing mode with the plasma frequency $\omega_{\mathrm{p}}$ : 


$$
\omega_{p}=\left(K \beta^{2} c^{2} / 2 r^{2}\right)^{1 / 2} \text { where } K=\frac{q I}{2 \pi \varepsilon_{0} \gamma^{3} \beta^{2} m c^{3}}
$$

and $\mathrm{r}$ is the beam radius. In the case simulated, in fig. $1 \omega_{\mathrm{p}}$ is about $51 \mathrm{MHz}$. In these conditions Landau damping can occur. In fact, there is a harmonic excitation given by the breathing mode oscillation of the beam envelope and a large set of oscillators, the beam particles oscillating with a large betatron frequency spectrum. From that figure, it can be noticed that the coherent oscillation energy is transferred to the incoherent oscillations of the beam particles. During the damping of the breathing oscillation there is a slight increase of the total emittance variation. At the end, we can say that the energy involved in this process is dissipated from a coherent motion to the very high number of degrees of freedom of the system. When this damping process is finished, the beam reaches the matched size and no further increase of emittance is observed.
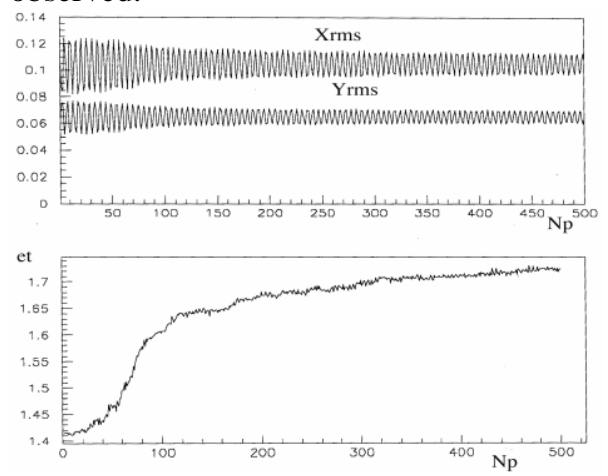

Fig. 2 Transverse rms beam size values $v s$ cell period numbers $(\mathrm{Np})$ for the case $\sigma / \sigma_{0}=0.29(\mathrm{I}=200 \mathrm{~mA})$.

In fig. 2 are shown simulation results with an increased space charge tune depression, $\sigma / \sigma_{0}=0.29$. In this case, it can be noticed that only some part of the beam particles is involved in the Landau damping process. In fact, now the plasma frequency is about $56 \mathrm{MHz}$ and the lowest betatron frequency is about $16 \mathrm{MHz}$. For this reason the part of the beam particles that have betatron frequencies too far from the plasma frequency interact very weakly with the coherent oscillation and then the breathing mode oscillation is only partly damped. In fig. 3 is shown the case of emittance dominated beam $\left(\mathrm{Kr}^{2} / 4 \varepsilon<<1\right)$ where no energy exchange occurs between the breathing mode and the betatron oscillations and emittance remain constant.

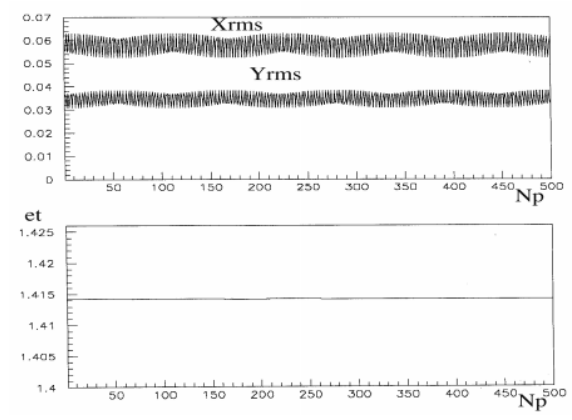

Fig.3 Transverse rms beam size values $v s$ cell period numbers $(\mathrm{Np})$ for the case $\sigma / \sigma_{0}=0.89(\mathrm{I}=0.5 \mathrm{~mA})$.

The test particle trajectories considered in ref.[5] to detect the chaotic motion which is responsible of beam halo generation, showed only coupling between the transverse planes but remained confined inside their original positions. Those test particles started from near the beam core, where rms oscillations, due to mismatching, occur but through the damping the beam readjusted itself at matching conditions very soon. Maybe, considering, in those simulations, a larger number of test particles some chaotic trajectories had could be found.

Chaotic motions have been found in the test particle trajectories considered for the case of a space charge dominated beam with $\sigma_{0}>90^{\circ}$ where envelope instabilities occur [11]. In fig. 4 are shown trajectory results for those test particles starting from near the beam core at a position around $x=0.2 \mathrm{~cm}$ (the same of ref.[5]). It can be noticed that, although the shown test particles initial conditions differ only of few microns they have completely different phase trajectories (chaotic behavior). Furthermore the chaotic test particles oscillate between $0.2-0.5 \mathrm{~cm}$ originating a beam halo size greater than $0.5 \mathrm{~cm}$.
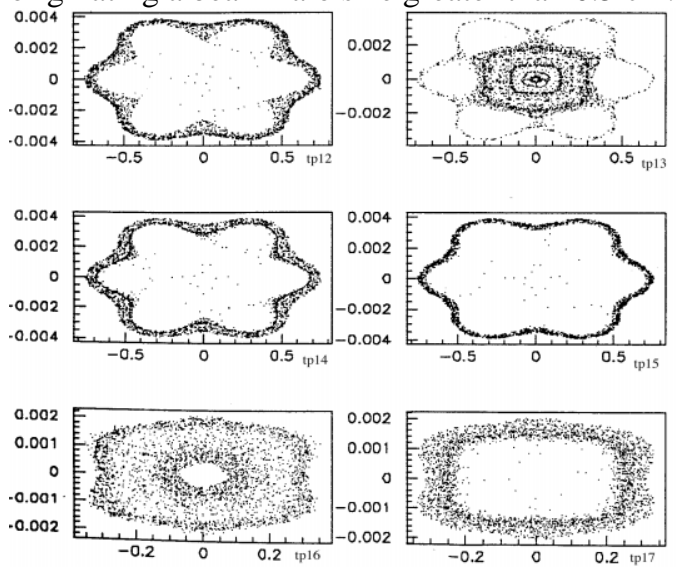

Fig.4 Chaotic test particle trajectories in phase space $\mathrm{xx}$ '.

\section{CONCLUSIONS}

The fast damping of the breathing mode oscillation occurring in mismatched beam space charge dominated can be ascribed to a Landau damping mechanism of stabilization. No test particle with chaotic motion has been found when fast damping of mismatched oscillation occurred.

\section{REFERENCES}

[1] C. Rubbia et al., CERN/AT/95-44(ET)

[2] C. Rubbia et al., CERN/AT/95-53(ET)

[3] R. A. Jameson, G. P. Lawrence and S. O. Schriber, Proc. PAC'93

[4] J. Lagniel, NIM in Phis. Res. A345 (1994) 405.

[5] V. Variale, submitted to NIM in Phis. Res. A

[6] J. Strukmeier, GSI-ESR-03 report.

[7] J. L. Laclare, CAS CERN85-19, Vol. II

[8] J. Landau J. Phys. USSR, 10, 25, 1946

[9] A. Hoffmann, CAS CERN95-06, Vol. I

[10] T. P. Wangler, AIP Conf. Proc. 377

[11] I. Hofmann, App. Char. Part. Optics 13C, ed. A. Septier (1983) 